\title{
Form and function
}

Because of our familiarity with the Watson-Crick form of DNA, we often overlook the fact that DNA can adopt other biologically relevant structures. Differences in the DNA structure in the promoter region of the oncogene MYC have previously been shown to affect its transcription. Analysis of the structure of this DNA region, in combination with a smallmolecule inhibitor of MYC transcription, provides new insights into the development of transcription-based anticancer drugs.

Within the promoter region of MYC is a nucleasehypersensitive element known as NHE III ${ }_{1}$, which controls up to $90 \%$ of $M Y C$ transcription. This region contains a pyrimidine-rich and a purine-rich strand, and is thought to be able to form alternative DNA structures. In particular, a purine-rich 27-nucleotide stretch that contains six guanine tracts is thought to form multiple guanine (G)-quadruplex structures. G-quadruplexes consist of successive layers of two or more G-tetrads to form a box-like structure with a central cavity. The G-tetrad consists of four guanines arranged in a cyclic, hydrogen-bonded square planar alignment (see accompanying picture). Substitution of these guanines with adenines disrupts this structure and increases MYC transcription, whereas stabilization of the G-quadruplexes by the small molecule ligand TMPyP4 represses $M Y C$ transcription.

Anh Tuân Phan and colleagues have resolved the structure of a 24-nucleotide, 5 guanine tract sequence $(\mathrm{Pu} 24)$ from the MYC NHE III ${ }_{1}$ element in a potassium-ion solution. The use of five guanine tracts has revealed new structural motifs not seen in previous structures that were resolved using four guanine tracts. By making a series of mutations based on this structure, the authors have identified novel motifs that are important for the folding and formation of this G-quadruplex. Importantly, the structural analysis of the interaction between the G-quadruplex and four different ligands has shown that all four bind on top of the G-tetrad core, but that subtleties occur in the effect that these ligands have on the stability of the G-quadruplex. Indeed, the kinetically slow binding of the TMPyP4 ligand to the G-quadruplex particularly enhances the stability of the complex, explaining its capacity to reduce $M Y C$ transcription.

The authors conclude that the new structural motifs and folding principles described for $\mathrm{Pu} 24$ will aid the understanding of other G-quadruplex structures. Such structures are evident in potential anticancer targets, such as the promoters of other oncogenes, and in telomeres.

Nicola McCarthy

Q) References and links ORIGINAL RESEARCH PAPER Phan, A. T. et al. Small-molecule interaction with a five-guaninetract G-quadruplex structure from the human MYC promoter. Nature Chem. Biol. 1, 167-173 (2005)
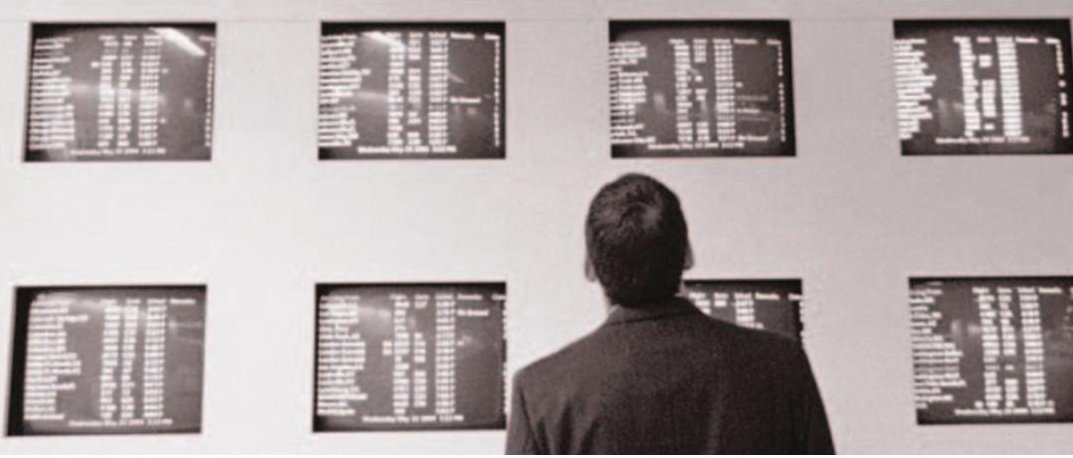

METASTASIS

\section{Destination control}

The heterogeneous nature of breast tumours results in widely divergent metastatic behaviour. Recently, it has been possible to correlate certain tumour-gene-expression signatures with either good or poor patient prognosis. Massagué and colleagues have taken this classification one step further, using microarray analysis to identify genes that selectively mediate the metastasis of breast cancer cells to the lung.

When breast cancers metastasize, they frequently spread to either bone or the lungs, through unknown mechanisms of tropism. Massagués group investigated this tropism by carrying out gene-expression analysis on breast cancer cells that had been selected for their ability to metastasize to the lung in mice. When the authors compared the transcriptomes of breast cancer cell lines with varying metastatic properties, they identified 54 candidate genes that the authors proposed were involved in either baseline lung-metastatic functions or lung-restricted, specialized functions that conferred lung-metastatic virulence.

In particular, high expression levels of genes encoding the celladhesion molecule SPARC, the cell-adhesion receptor VCAM1 and matrix metalloproteinase 2 (MMP2) were detected in highly metastatic cells. Other genes - including ID1, which encodes a transcriptional inhibitor of cell differentiation and senescence, and MMP1 - increased in relation to the lung-metastatic ability of the cell line.

When the authors overexpressed combinations of these genes in the parental cell line, they found that certain combinations conferred high levels of lung-selective metastatic activity. Furthermore, reducing the expression of some of these genes using RNA interference could decrease the lung-metastatic activity of the selected cells. Therefore, these genes are both markers and functional mediators of lung-selective metastasis.

To obtain clinical validation of their experimental findings, the authors analyzed expression levels of these genes in a cohort of human primary breast cancers. Patients with tumours expressing these genes had a poor lung-metastasisfree survival time, but expression of these genes did not correlate with bone-metastasis-free survival. Further analysis revealed that several of these lung-metastasis genes also facilitated breast tumorigenicity, which would explain how cells expressing these genes can be selected for in the primary tumour. Expression of other genes that are found in highly metastatic cells, however, is rare in primary tumours and does not significantly promote their growth, but once these rare cells reach the lung these genes are strongly selected for.

Lesley Cunliffe

Q) References and links ORIGINAL RESEARCH PAPER Minn, A. J. et al. Genes that mediate breast cancer metastasis to the lung. Nature 436, 518-524 (2005) FURTHER READING Weigelt, B., Peterse, J. L \& van't Veer, L. J. Breast cancer metastasis: markers and models. Nature Rev. Cancer 5, 591-602 (2005)

WEB SITE

A 4-strand parallel G-quadruplex with 3 guanine tetrads (yellow squares)
Joan Massagué's lab: http://www.mskcc.org/ $\mathrm{mskcc/html/10614.cfm}$ 